\title{
Distributed Multi-weight Data-gathering and Aggregation Protocol in Fleet Wireless Sensor Networks: Optimal and Heuristic Algorithms
}

\author{
Chao Gao * Guorong Zhao Shuang Pan Jianjun Zhou \\ Department of Control Engineering \\ Naval Aeronautical and Astronautical University, Shandong 264001, P.R. China \\ * Corresponding author's Email: gaochao.shd@163.com
}

\begin{abstract}
Clustering Algorithm is a kind of key technique used to reduce energy consumption, which can increase network scalability and lifetime. The main goal of this research is concerning protocols to contain in-motion sensor nodes in large-scale topologies, and constitute a fleet wireless sensor network which can assistant in-flight localization and alignment for carrier-based aircraft. In this paper, we abstract network model according to specific conditions of F-WSN, and select performance indicators to evaluate it. A distributed multiple-weight data gathering and aggregation protocol (DMDG) is proposed, which contains all-sided active clustering scheme and realizes long-range realtime communication by tacical data link under a TDMA/CSMA channel sharing mechanism. An analytical paradigm facilitating the most appropriate choice of the next relay is proposed. Experimental results have shown that DMDG scheme can balance the energy consumption and extend the network lifetime notably, outperform LEACH, PEACH, and DEEC in terms of network lifetime and coverage rate, especially in sparse node density or anisotropic topologies.
\end{abstract}

Keywords: Wireless sensor networks; Clustering algorithm; Data gathering; Lifetime; Carrier-based aircraft

\section{Introduction}

With the development of the very-large-scale integration(VLSI), micro-electro-mechanism system (MEMS) and networking technology, wireless sensor networks (WSNs) have attracted more attention lately[1]. The envisioned applications of wireless sensor networks range widely, such as environment monitoring, disaster surveillance, millitary target tracking, and medical treatment, among others. Therefore, technics related to wireless sensors are under active research and development in recent years.

The finial purpose of this paper is to design a fleet wireless sensor network (F-WSN) which can meet the need of localization and in-flight alignment for carrier-based aircraft. There are many challenges when designing and deploying F-WSN in those conventional WSN protocol, and an unavoidable one is how to make full use of the limited sensor resource to provide a long and precise service.
Because of the network nodes are sparse and in-motion, a network has to be designed to utilize its sensing resource efficiently. Many efforts have been devoted to sensing resource efficiency of wireless sensor networks, most of which proposed new protocols to preserve sensing resource as much as possible during the process of collecting data[2,3]. Many researchers focused on next-hop selection strategies, which made one-hope neighboring nodes or multiple link candidates consume sensing resource evenly for transmitting data[4,5]. There are already some successful examples of fleet networks, including joint tactical information distribution system (JTIDS), MIDS etc.With the assistant of tactical data link(Link-16 or TTNT), the network can provide precise navigation information including position (or relative position), velocity (or relative velocity), heading angle, and system clock. However, these networks were designed on the early ad-hoc technology, which was unperfect and had its inherent defects. So it is necessary to design an all-purpose F-WSN protocol frame based on the 
up-to-date progress of WSN theory.

The remainder of this paper is structured as follows. Section 2 surveys related work, including basic concepts and model. Section 3 introduces the proposed DMDG protocol. Section 4 discusses the protocol performance. Section 5 concludes the paper.

\section{Related work}

Work in the area of cluster-based wireless sensor networks is quite extensive, with energy efficiency and scalability being the main focus of many of the clustering protocols proposed so far[6-10].Similarly, much work has been done on sensor activation protocols, which focus on selecting a subset of the active sensor nodes that are sufficient to satisfy the network's coverage requirments. Compare to traditional WSNs, fleet sensor network has special characters in a couple of aspects, including high-powered links, high speed nodes, sparse and very-large-scale working range etc. In this section, we discuss the related work that has been done take account of these pionts.

\subsection{Network model}

In this subsection, we first introduce the characteristics of WSN and then define the network model of WSN. We model the WSN as a graph, $G=\left(C, E, R_{c}\right)$, which consists of a set of mobile nodes, $C=\left\{C_{1}, C_{2}, \cdots ; C_{m}\right\}$, and a set of wireless links, $E$, each sensor node consists of components of sensing, computing and wireless transmission. Assume that each node has the same transmission radius $R_{t}$ and the same sensing radius $R_{s}$. The notations used in this paper are defined in Table 1.

Before discussing the nuture of this problem, we give the following assumptions for the feasibility of the F-WSN, which include monitor area, transmission channel and communication environment.

Assumption 1 (Monitor area). The monitor area of F-WSN $A_{c}$ is a cylinder, which is equivalent with the tradition definition of defense area.

$$
A_{c}=\left\{(x, y, z) \mid x^{2}+y^{2} \leq R_{\mathrm{cov}}, 0 \leq z \leq h_{\mathrm{cov}}\right\}
$$

where $R_{\text {cov }}$ is monitor radius, $h_{\text {cov }}$ is monitor height.

Assumption 2 (Signal link). The transmission path adopts tactical data link. To meet the requirement of real-time performance and reliability, $R_{c}$ is defined as $1 / 3-1 / 5$ ratio to the effective tansmission radius of the link, and assumped that the bit error rate (BER) is invariable.

Table 1. Notation and definition in F-WSN

\begin{tabular}{ll}
\hline Notation & Definition \\
\hline$A_{c}$ & Work area of the fleet wireless sensor \\
$C=\left\{C_{1}, C_{2}, \cdots, C_{m}\right\}$ & network \\
$E=\left\{(i, j) s(i, j) \leq 2 R_{c}\right\}$ & Set of F-WSN mobile nodes \\
$C_{M s g} \in G$ & Set of wireless links \\
& Structure message of F-WSN nodes \\
$\operatorname{Link}_{j}^{i}(\tau)$ & Signal links between node $i$ and $j$ \\
$T_{y p e_{k}^{i}}$ & at time $\tau$ \\
& Type of the node $i$ in cluster $k$, \\
& Type ${ }_{k}^{i} \in\{$ Memb,CH, agent,NR,toCH,toNR\} \\
$R_{m s g}(i)$ & Maximum transmission range of \\
$R_{c}(i)$ & node $i$ \\
$T_{\text {life }}(i)$ & Sensing radius of node $i$ \\
\hline
\end{tabular}

Assumption 3 (Communication environment). The statistical property of the NLOS propagation accord with exponential distribution, while the time-delay accord with Bernoulli distribution. The coupling and interfere in electro magnetic environment are mutually independent white noise with zero means.

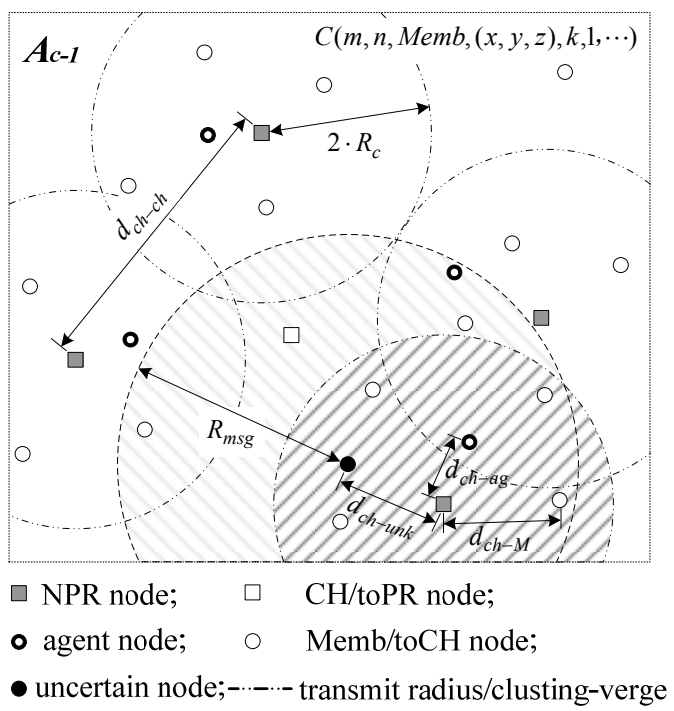

Figure.1 Sketch map of F-WSN model

Fig. 1 is an example of F-WSN, in which the type of area coverage $A_{c-1}$ is considered. $A_{c-1} \subset A_{c}$, and $d_{c h-c h}, d_{c h-M}, d_{c h \text { unk }}, d_{c h-a g}$ represent the distance between neighbor cluster-heads, cluster-head to cluster-member, cluster-head to uncertain node, and 
cluster-head to agent node respectively. $2 R_{c}$ is the cluster radius, and $R_{m s g}$ is the searching radius of uncertain node. Each interested grid has at least one sensor node within it or the grid can be sensed by neighbor sensors.

\subsection{Evaluating Indicator Used in F-WSN}

\section{(1) Coverage relavant model}

Coverage is an important issue of WSNs and is narrowly related to energy saving, connectivity, network reconfiguration, etc[11-14]. The problem of how well a given area $A_{c}$ can be monitored by the F-WSN which is known as coverage, can be described by two aspects. The following is the main methods we adopt to assess coverage, which comprise 3D $k$-coverage model and the NSC (necessary and sufficient condition).

Definition 1. (3D $k$-covered problem). Given a set of $N$ sensors in area $P$, the radius of sensor node is $R_{c}$, if there is $C_{i 1}, \cdots, C_{i k} \in\left\{C_{1}, \cdots, C_{n}\right\}, k \geq 1$. In this case, every position $v$ in area $P$ can meet the following formula

$$
v \in \bigcap_{j=1}^{k}\left\{x \mid \operatorname{dist}\left(x, C_{i j}\right) \leq R_{c}\right\}
$$

Then area $P$ is $3 D k$-coverage by $\left\{C_{1}, C_{2}, \cdots, C_{n}\right\}$.

Theorem 1 is the necessary and sufficient condition used to determine the connected coverage set, which has been proved by Zhang $\mathrm{H}$ etal in literature [2].

Theorem 1. Given a set of $N$ sensors $C$ in three-dimensional area $P, C=\left\{C_{1}, C_{2}, \cdots, C_{N}\right\}$, the communi-cating radius of sensor node is $R_{c}$, the sensing radius of sensor node is $R_{c} \geq 2 R_{s} . \exists C_{i} \subset C$, $C_{i}=\left\{C_{i 1}, \cdots, C_{i k}\right\}$. If sensing area of $C_{i}$ can complete cover $P$, the set $C_{i}=\left\{C_{i 1}, C_{i 2}, \cdots C_{i k}\right\}$ is a connected coverage set.

\section{(2) Link Consumption Indicator}

Link quality is one of the key factors which affected the application of WSN technique. In this paper, we adopt tactical data link to extend it to solving the fleet-network-buildup problem, in which the following link consumption model is adopted.

For relatively short distances, the propagation loss is modeled as being inversely proportional to $d^{2}$, whereas for longer distance, the propagational to $d^{4}$. Power control can be used to invert this loss by setting the power amplifier to ensure a certain power at the receiver[15-17]. Therefore, to transmit and to receive an $l$-bit packet in a distance $d$, the radio expends the following energy, respectively:

$$
\begin{aligned}
& E_{T x}(l, d)=l \cdot E_{T x-\text { elec }}+E_{T x-\text { amp }}(l, d) \\
& = \begin{cases}l \times E_{\text {elec }}+l \times \varepsilon_{f t} \times d^{2} & d<d_{0} \\
l \times E_{\text {elec }}+l \times \varepsilon_{\text {amp }} \times d^{4} & d \geq d_{0}\end{cases} \\
& E_{R x}(l, d)=E_{R x-\text { elec }}(l)=l \times E_{\text {elec }}
\end{aligned}
$$

where $d_{0}$ is the free space model and multipath fading model. $E_{T x-e l e c}$ is the electronics energy and depends on factors such as digital coding, modulation, and filtering of the signal before it is sent to the transmit amplofier. The parameters $\varepsilon_{f t}$ and $\varepsilon_{a m p}$ depend on the required sensitivity and the noise figure of the receiver[12].

For the experiments decribed in this paper, we adopted the parameters of the radio chips similar to those in [6] to detemine the parameters values in Eqs.(1) and (2). Then we have the representative values of the parameters $\varepsilon_{a m p}=0.0013 \mathrm{pJ} / \mathrm{bit} / \mathrm{m}^{4}$; $E_{\text {elec }}=50 \mathrm{~nJ} / \mathrm{bit} ; \varepsilon_{f t}=10 \mathrm{pJ} / \mathrm{bit} / \mathrm{m}^{2}$.

\section{DMDG protocol}

In this section, we present the distributed multiple-weight data gathering and aggregation protocol (DMDG) after describing the nerwork model and evaluating indicator adopted, which consists of three parts as described in the following subsection.

\subsection{Clustering algorithm}

The hierarchical clustered sensor network is composed of a number of clusters. The follows are some important definitions used in the clustering algorithm.

Definition 2. (Initial probability for cluster-head) .

$$
p_{\text {init }}=\beta \cdot I D_{i} / I D_{\max }+(1-\beta) \cdot G D O P / G D O P_{\max }
$$

where $I D_{i}$ is the initial priority of sensor node $i$, $I D_{\max }$ is the maximum priority within the spheric radius $r_{c}, G D O P_{i}$ is the GDOP (geometric distribution of position) of sensor node $i, G D O P_{\max }$ is the optimum GDOP within the spheric radius $r_{c}$, and $\beta$ is a self-adapting weighing factor.

Definition 3. (Electing-factor for cluster head, $\mathrm{EFCH})$.

$$
W_{i}=\alpha \cdot\left(E_{i j} / \bar{E}^{r}\right)+(1-\alpha) / 2 \cdot \frac{d_{\max }-d_{t o B S}^{i}}{d_{\text {max }}-d_{t o B S}}
$$




$$
+(1-\alpha) / 2 \cdot G D P_{k}
$$

where $\alpha$ is a self-adapting weighing factor, $\alpha=1 /(2+\beta),\left(\beta=E_{i j} / \bar{E}^{r}\right) . \quad E_{i j}$ is the mean energy value of communicated comsumption between sensor node $C_{i}$ and other nodes in the same cluster, $\bar{E}^{r}=E_{\text {total }}(r) / m . d_{\text {toBS }}^{i}$ is the distance between $C_{i}$ and cluster-head node $\mathrm{CH}_{k} \cdot d_{t o B S}$ is the distance between $\mathrm{CH}_{k}$ and the cluster-center, $d_{t o B S}=\sum_{i=1}^{m} d_{t o B S}^{i} / m . G D P_{k}$ is the GDOP of cluster-head node $\mathrm{CH}_{k}$.

Table 2. Main clustering procedure pseudo-code

Input: Sensing range $R s$, maximum transmission range $R t$,sensor nodes set $V$, and $\varepsilon_{0}$.

Output: agent, $C_{C H}, C_{P R}$.

1. for all $u, v \in V(\mathrm{G}), \mathrm{i} \in N E W(\mathrm{G})$ do

2. while round $==1 \quad / / \mathrm{F}-\mathrm{WSN}$ initialization

3. $\quad \mathrm{C}[v] .\{$ ID, Type $\} \leftarrow$ sensor[i]. \{PRI,Power $\}$

4. Subprogram GetFirst_CHs();

5. end-while

6. while round $==0 \quad / / F-W S N$ regular circuit

7. $\quad$ if $($ C.new $\cap V(\mathrm{G})=\varnothing \| \mathrm{C}[\mathrm{i}] \cap V(\mathrm{G})=\varnothing)$

8. Subprogram NEW_DEAD();

9. $\quad$ elseif $($ toCH $[v] \cup$ toAgent $[v] \neq \varnothing)$

10. Subprogram RENEW_agent();

11. Subprogram RENEW_CHs();

12. $\mathrm{CHs}[v] \leftarrow \mathrm{NEW} \_\mathrm{CHs}[v] ; \mathrm{AGs}[v] \leftarrow \mathrm{NEW} \_\mathrm{AGs}[v]$;

13. end-if

14. $\mathrm{CHs}[u] \leftarrow \mathrm{CHs}[v] ; \operatorname{AGs}[u] \leftarrow \mathrm{AGs}[v]$;

15. CHs. \{\}$\rightleftharpoons$ Ags. \{\} ;

16. BroadcastMsg(Ags $[u]$. \{ID,nbn,Level,CP $\})$;

17. BroadcastMsg $(\mathrm{CHs}[u]$. $\{\mathrm{ID}, \mathrm{nbn}$, Level, $\mathrm{CP}\})$;

18. while $($ toPR $[v] \neq \varnothing$ \& round $==0$ )

19. Subprogram RENEW_PRs();

20. BroadcastMsg(C[i]. $\{$ ID,nbn, Level, CP $\})$

21. end-while

22. BroadcastMsg(C[i]. $\{\mathrm{ID}, \mathrm{nbn}$, Level,CP $\})$;

23. end-for

Definition 4. (Correlation among sensor-nodes). $\forall C_{i}, C_{j} \in G(V), \exists R\left(C_{i}, C_{j}\right) \in R^{+}, i \neq j$, if $\left\|D\left(C_{i}, C_{j}\right)\right\|$ $\leq r,\left\|R\left(C_{i}, C_{j}\right)\right\|=1$; else if $\left\|D\left(C_{i}, C_{j}\right)\right\|>r,\left\|R\left(C_{i}, C_{j}\right)\right\|$ $=n+1$, where $n$ is the sensor number beween $C_{i}$ and $C_{j}, r$ is the average 1-hop distance, then
$R\left(C_{i}, C_{j}\right)$ is called as the correlation between node $C_{i}$ and $C_{j}$.

Now we describe our cluster formation algorithm in detail. The algorithm consists of three stages. In the initial stage, F-WSN nodes initiates the clustering procedure(Lines $2-5$ ), round $=1$, fleet sensors sensor $[i]$ join into the network and obtain their own IDs and Types, which are proportional to $P R I$ and power of their link-terminal, then run the subprogram GetFirst_CHs() to elect the first cluster-head and agent, which is direct ratio to $I D$ and Type.

When F-WSN is formed, round $=0$, the program of regular circuit(Lines 7-21) will be performed. In the beginning of every cycle, cluster-heads should judge whether there are new-comming requisitions C.new or new-dead node, and judge whether there are new requisition for cluster-head $t o C H[v]$ and for agent toAgent $[v]$, then executes relative treating subprograms NEW_DEAD(), RENEW_agent(), RENEW_CHs(), in which the first one is related to IDs and Types, and the last two subprograms are mainly determined by $p_{\text {init }}$ and $W_{i}$ which is defined in Definition 2 and Definition 3. When $\mathrm{CHs}[i]$ is selected as a cluster head, it broadcasts message $M s g()$ to all other nodes to indicate its identity and adjust TDMA format. The agent and cluster-head also communicate every cycle to backup information to preserve robustness and stability of the network. The procedure also handling the request for reference nodes $R E N E W \quad P R s()$ to realize renew of the network and assure load-balance of the clustering protocol.

\subsection{Data aggregation and communication}

This subsection presents the data aggregation mechanism and the communication mode among F-WSN nodes. The data transmitted among network members mainly has four portions, including the communication between reference nodes $C_{P R}$ and cluster-head $C_{C H}$, cluster-head $C_{C H}$ and the neighbor cluster-head, cluster-head $C_{C H}$ and the cluster member $C_{C M}$, and cluster member $C_{C M}$ and the 2-hop neighbor node. Each transmission process has its own packet mode, including broadcast, data-centric, local transmission, and multicast. The data packets have an unified scheme, main portions of which are shown in Table 3. Mainly composed of synchronous head, node's structural information $C_{M s g}$, sphere coordinate coefficient $T S_{C A}$, relative velocity $V_{C}$, heading angle $\phi_{C}$, system time $t_{A}$ 
etal. The received variables should accord with transmission demand and uniform formats, and the redundant information should be neglected.

Table 3. The format of data packet

\begin{tabular}{lcccccc}
\hline Packet & head & $C_{M s g}$ & $T S_{C A}$ & $V_{C}$ & $\phi_{C}$ & $t_{A}$ \\
\hline NR-CH & $\sqrt{ }$ & $\sqrt{ }$ & $\sqrt{ }$ & - & $\sqrt{ }$ & $\sqrt{ }$ \\
CH-CH & $\sqrt{ }$ & $\sqrt{ }$ & - & - & - & $\sqrt{ }$ \\
CH-CM & $\sqrt{ }$ & $\sqrt{ }$ & $\sqrt{ }$ & - & - & $\sqrt{ }$ \\
CM-CM & $\sqrt{ }$ & $\sqrt{ }$ & - & $\sqrt{ }$ & $\sqrt{ }$ & - \\
\hline
\end{tabular}

The four packet formats which are put forward in Table 3 have their appointed operation occasion and objects. Then we analyze them detailedly as follow.

- NR-CH: The data packet between reference node and cluster-head node $C_{C H}$ has three kinds of process: (1) the position reference nodes $C_{P R}$ broadcast global coordinate information and the network distributing variable to cluster-head nodes $C_{C H}$; (2) the time reference nodes $C_{T R}$ broadcast system time $t_{A}$ to $C_{C H}$ and other network member; (3) $C_{C H}$ uploads its own sensing parameter and cluster-member dynamic information to neighbor $C_{P R}$, which can be used for the next reference nodes' election.

- CH-CH: This transmiting process is to modify locali-zation information for each other, which is used to define cluster verge. It is also used to acquire $I D$ of neighbor cluster-head node, which can be a relay node for multi-hop transmission.

- CH-CM: In this process, cluster-head $C_{C H}$ collects sensing information from its own cluster members $C_{C M}$, which is used for data aggregation and fusion. Then $C_{C H}$ transmits system time $t_{A}$, transition matrix $T S_{C A}$, and other information to $C_{C M}$ under a TDMA channel sharing mechanism.

- CM-CM: In this process, cluster-head $C_{C H}$ broadcast sensing information to neighbor cluster-heads in its own TDMA-based time slot.This information is mainly used to crrect navigation parameters including position, velocity(or relative velocity), and heading angle. Other information is calculated into evaluating indicators, which are difined in section 2.2 and 3.1 , for the next cluster-head election.

\subsection{Three-dimensional routing algorithm}

When the network is clustered, specific methods for intracluster and intercluster communications depend on applications. For intracluster communication, the nodes can directly send data to the cluster head using time-division multiple-access (TDMA) schedule, just as in LEACH[7].

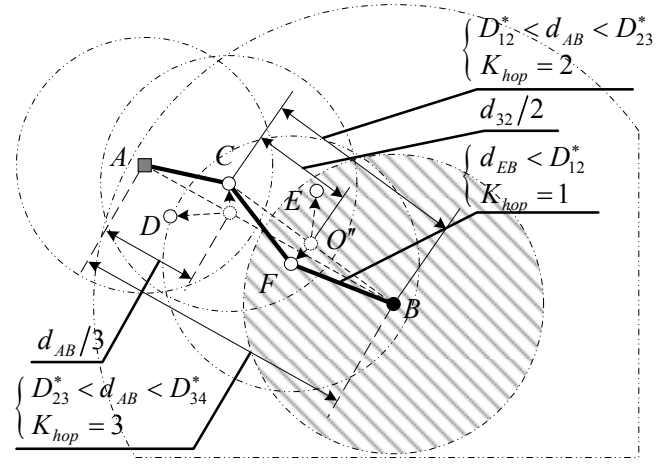

Figure.2 Geographic routing algorithm in F-WSN

As shown in Fig.2, when node B broadcasts a packet, all the one-hop neighbors receive that packet. In order to avoid recipient ambiguity in a local broadcast, the incoming label of a node must be unique at least in its 2-hop neighbors. In the label assignment phase, the cluster-head must execute an algotithm to ensure that each node is allocated a unique incoming label.A 3-D heuristic routing algorithm is proposed for this purpose, which can be expatiated in the following example.

- Step 1: When node $A$ receive the request packet from the node $B$, firstly, estimate whether the distance between node $B$ and $A$ $\left(d_{A B}\right)$ satisfy the responsing condition, $d_{A B} \leq 2 \cdot R_{c}$, and calculate the number of optimum hops ( $\left.K_{\text {hop }}\right)$ if the condition is satisfied, if not, ignore the request. As shown in Fig.2, $K_{\text {hop }}=3$.

- Step 2: Node $A$ computes the first trisectional point $O^{\prime}$ between $A$ and $B$ based on geographic position got from the request packet, then confirm point $O^{\prime}$ as the ideal next-hopposition.

- Step 3: Node $A$ compares all the distance between one-hop neighbor (node $C$ and $D$ ) and point $O^{\prime}$, then choose the next-hop node with the following equation, $H o p_{i}=\min _{i}\left\{P_{i}-P_{o p t}\right\}$, in which $i$ is $I D$ of one-hop neighbors. So in the case of Step 2, the next-hop neighbor of node $A$ is $C$, which obtain the information pactet.

- Step 4: Node $C$ repeats the steps $(\operatorname{step}(1),(2)$, 
(3)), and confirm the next-hop relay $E$, where $K_{\text {hop }}=2$, then the node $F$ obtain its next-hop relay $B$, where $K_{h o p}=1$. So the route is confirmed, and the information transmitted through the route A-C-F to $B$, the routing procedure is over.

\section{Performance evaluation and simulation}

To evaluate the performance of our scheme and compare it to both the association sponsor and central angles method, a set of simulations have been carried out, which are described in the following section.

\subsection{Simulation set-up}

The proposed DMDG protocols were evaluated by extensive computer simulations by Matlab 7.1 and compared with LEACH[7], DEEC and PEACH The performance compared includes network lifetime, costs associated to clustering and backbone formation, as well as the properties of generated clusters[19-24]. The simulations are conducted with $R_{\text {cluster }}=2 \cdot R_{s}$, and main simulation parameters are summarized in Table 4 . We assume that all the messages received from the cluster members can be aggregated into a single message.

Table. 4 Main Simulation Parameters

\begin{tabular}{|c|c|}
\hline Parameters & Values \\
\hline Network size $(R \operatorname{cov}, h)(\mathrm{km})$ & 20,2 \\
\hline The number of sensor nodes & 30 or 50 \\
\hline Sensing range $(R c)(m)$ & $5000 \sim 10000$ \\
\hline $\begin{array}{l}\text { Maximum transmission range } \\
(\text { Rtmax })(m)\end{array}$ & 15000 \\
\hline Time interval for reporing data & $30 \mathrm{~s}$ \\
\hline MAC protocol & TDMA/CSMA \\
\hline Radio frequency $(\mathrm{MHz})$ & $960 \sim 1206$ \\
\hline$E_{\text {elec }}$ in the energy $\operatorname{model}(\mu \mathrm{J} / \mathrm{bit})$ & 1.16 \\
\hline$d_{0}$ in the energy $\operatorname{model}(\mathrm{km})$ & 10 \\
\hline$\varepsilon_{f t}$ in the energy model $\left(\mathrm{pJ} / \mathrm{bit} / \mathrm{m}^{2}\right)$ & 10 \\
\hline$\varepsilon_{\text {amp }}$ in the energy model $\left(\mathrm{pJ} / \mathrm{bit} / \mathrm{m}^{4}\right)$ & 0.0013 \\
\hline
\end{tabular}

\subsection{Performance result and analysis}

Fig. 3 presents the average power consumption per node obtained by using LEACH, DEEC and DMDG, and average number of hops versus the number of nodes when the maximum transmission range $R_{m s g}=15000 m$, and the number of the node increase from 5 to 50. It is clearly in Fig.3(a) that DMDG always achieves the lowest power consumption and a relative stable hop number, $N_{h o p} \approx 2$, which is gradually increasing in LEACH and DEEC. The average power consumption using DMDG can be as low as 0.637 when compared to LEACH, and 0.824 when compared to DEEC. The stability in average number of hops results in stable of end-to-end delay, this can prove to be an advantage since it is convenient for estimating end-to-end delay and guarantee a data synchronism.

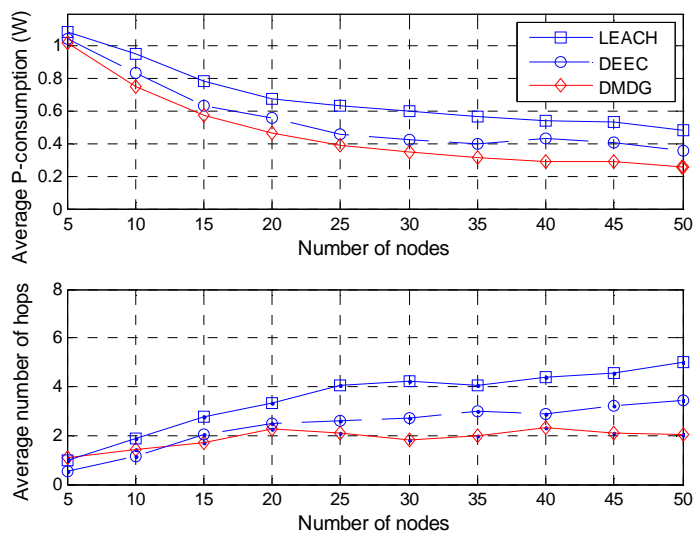

Figure.3 Average power consumption and average number of hops vs. the number of nodes, $N$

Fig. 4 presents the lifetime and stable periods of the related LEACH, DEEC, PEACH and DMDG protocol. The result is closely related with the topocol balance of the F-WSN. In the case of networks using DMDG with the maximum transmission range $R_{m s g}=5000 \mathrm{~m}$, the proportion of dead nodes(renew for mission) is $26 \%$ in lifetime, and increase slowly in stable period(about $51 \%$ in the fourth day), which is much better than other protocols.
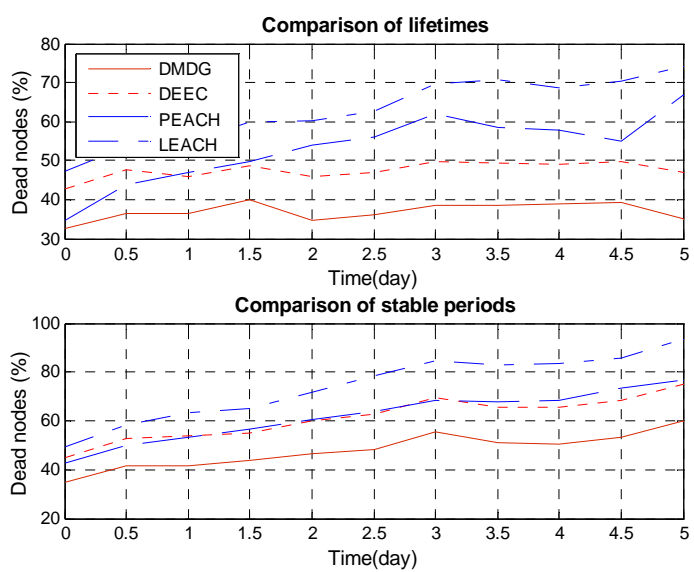

Figure.4 Comparison of lifetime and stable periods between LEACH, PEACH, DEEC and DMDG 
Fig. 5 shows the coverage rate versus proportion of dead nodes in different sensing range $R_{c}=5,8,10 \mathrm{~km}$. Comparing the tendency curves of coverage rate with the increasing dead node, in which adopt $3 D$-coverage model introduced in Definition.1, $k=2$, and consider the interfere from in-motion nodes. The result shown that DMDG can achieve a full 2-coverage if the number of the dead nodes $n_{\text {dead }} \leq 11$ when $R_{c}=5 \mathrm{~km}, n_{\text {dead }} \leq 20$ when $R_{c}=8 \mathrm{~km}$, and $n_{\text {dead }} \leq 29$ when $R_{c}=10 \mathrm{~km}$, this conclusion approve that the DMDG protocol can guarantee a high coverage environment in a fleet network with low density in-motion nodes.

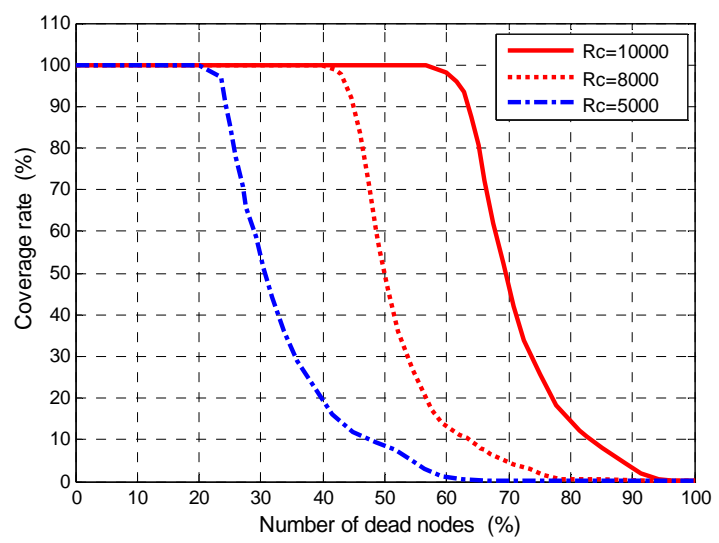

Figure.5 Coverage rate vs. number of the dead nodes

$$
\left(R_{c}=5,8,10 \mathrm{~km}\right)
$$

\section{Conclusion and future work}

This paper presented a new clustering and routing scheme, the distributed multiple-weight data gathering and aggregation protocol (DMDG), which works under long distance sparse F-WSN and can efficiently guarantee a long stable coverage rate through a unit circle test. Simulation results also show that DMDG protocol can significantly extend the network lifetime when compared with other approaches. The F-WSN with DMDG protocol is equally applicable to the cases of three-demensional localization and in-flight alignment for carrier-based aircraft. The detail performance evaluation of these cases will be resolved in the following research.

\section{Acknowledgments}

This work is supported by the National Defense Previous Research Foundation of China (Grant No. 51309060401). The authors express sincere apprecia-tion to the reviewers of this paper for their helpful recommendations.

\section{References}

[1] Chong C Y, Kumar S P. Sensor networks: evolution, opportunities and challenges. Proc.of the IEEE, 2003,91(8), pp.1247-1256.

[2] Zhang H, Hou JC. Maintaining sensing corerage and connectivity in large sensor networks. Ad Hoc \& Wireless Networks, 2005,1(1), pp.89-124.

[3] Stanislava Soro, Wendi B. Heinzelman. Cluster head election techniques for coverage preservation in wireless sensor networks. Ac hoc networks, August, 2008, pp.955-972.

[4] Costa J A, Patwari N, and Hero A O. Distributed weighted-multidimensional scaling for node localization in sensor networks. ACM Transactions on Sensor Networks, 2006,2(1), pp.39-64.

[5] Khaled Matrouk, Bjorn Landfeldt. KETT-gen: A globally efficient routing protocol for wireless sensor networks by equalising sensor energy and avoiding energy holes, Ad hoc networks, July 2008, pp.514-536.

[6] Chao Gao, Guorong Zhao, Shuang Pan. Research on network-assistant alignment for carrier-based aircraft based on wireless sensor network. Acta aeronautica et astronautica sinica. unpublished.

[7] Y. Yu, R. Govindan, and D. Estrin, Geographical and energy aware routing: a recursive data dissemination protocol for wireless sensor networks.May, 2001.

[8] Sangho Yi, Junyoung Heo, Yookun Cho etal. PEACH: Power-efficient and adaptive clustering hierarchy protocol for wireless sensor networks. Computer communications, May,2005, pp.2842-2852.

[9] Xiaorong Zhu, Lianfeng Shen, and Tak-Shing Peter Yum. Hausdorff clustering and minimum energy routing for wireless sensor networks. IEEE transactions on vehicular technology. vol. 58, no. 2, February, 2009, pp. 990-996.

[10]L.Galluccio, A.Leonardi, G. Morabito etal. A MAC/ Routing cross-layer approach to geographic forwarding in wireless sensor networks, Ad hoc networks, February, 2007, pp.872-884.

[11]Jamal N, Al-Karaki, Raza Ul-Mustafa etal. Data aggregation and routing in wireless sensor networks: optimal and heuristic algorithms, Computer networks, December, 2008, pp.1-16.

[12]Azzedine Boukerche, Xin Fei. A coverage-preserving schme for wireless sensor network with irregular sensing range, Ad hoc networks, March, 2007. pp.1303-1316.

[13]REN Feng-Yuan, DONG Si-Ying, and HE Tao etc. A time synchronization mechanism and algorithm based on phase lock loop. Journal of Software, 2007,18(2), pp.372-380.

[14]Mihaela Cardei, Jie Wu. Energy-efficient coverage problems in wirless ad-hoc sensor networks[J]. Computer communications. 2006(29), pp. 413-420.

[15]Yan Jin, Ling Wang, Yoohwan Kim,etal. EEMC: An energy-efficient multi-level clustering algorithm for large-scale wireless sensor networks $[\mathrm{J}]$. Computer Networks, 2008(52):542-562. 
[16]Chee-Wei Ang, Chen-Khong Tham. iMST: A bandwidth-guaranteed topology control algorithm for TDMA-based ad-hoc networks with sectorized antennas[J]. Computer Networks, 2008(52):1675 $-1692$.

[17]Ben-Jye Chang, Jia-Bin Peng. On the efficient and fast response for sensor deployment in sparse wireless sensor networks[J]. Computer communi -cations. 2007(30), pp. 3892-3903.

[18]Yibin Yu, Xiaoning Xue, Xiaozhen Wang. Location discovery for three-Dimensional sensor-actor networks using alternating combination quadrilater -ation[J]. $20066^{\text {th }}$ Intermational Coference on ITS Teleconunuic\&ations Proceedings.2006, pp. 10211024.

[19]Shun-Yu Chuang, Chien Chen, Chang-Jie Jiang. Minimum-delay energy-efficient source to multisink routing in wireless sensor networks[J]. Signal Processing, 2007(87), pp. 2934-2948.

[20]Zhang H, Hou JC. Maintaining sensing corerage and connectivity in large sensor networks[J]. Ad Hoc \& Wireless Networks, 2005,1(1), pp.89-124.

[21]Song Ci, Mohsen Guizani, Hamid Sharif. Adaptive clustering in wireless sensor networks by mining sensor energy data[J]. Computer Communications, 2007(30), pp.2968-2975.

[22]Shun-Yu Chuang, Chien Chen, Chang-Jie Jiang. Minimum-delay energy-efficient source to multisink routing in wireless sensor networks[J]. Signal Processing, 2007(87), pp.2934-2948.

[23]Tuan Le, Wen Hu, Peter Corke etal. ERTP: Energy -efficient and reliable transport protocol for data streaming in wireless sensor networks[J]. Computer Communications, 2009(21), pp.3235-3251.

[24]Ameer Ahmed Abbasi, Mohamed Younis. A survey on clustering algorithms for wireless sensor networks[J]. Computer Communications, 2007(30): 2826-2841. 